\title{
LEAFY COTYLEDON1, FUSCA3 expression and auxin treatment in relation to somatic embryogenesis induction in Arabidopsis
}

\author{
Agnieszka Ledwoń · Malgorzata D. Gaj
}

Received: 6 September 2010/Accepted: 12 March 2011/Published online: 29 March 2011

(C) The Author(s) 2011. This article is published with open access at Springerlink.com

\begin{abstract}
The expression pattern of the LEC1 and FUS3 genes during somatic embryogenesis in Arabidopsis explants (immature zygotic embryos) induced in vitro was analysed, using Real-time quantitative PCR (qRT-PCR). The analysis revealed differential expression of $L E C 1$ but not FUS3 within a 30 day time course of somatic embryo development, and a significant auxin-dependent upregulation of $L E C l$ was found over the time course. In contrast to embryogenic culture, the level of LECl and FUS3 expression was noticeably lower in non-embryogenic callus of Col-0 and hormonal mutants (cbp20 and axr4-1) with low SE-efficiency. In addition, the expression profile of LEC1 and FUS3 was followed in the embryogenic culture derived from 35S::LEC2-GR explants. A significant increase of $L E C 1$ but not FUS3 activity was observed under LEC2 overexpression induced in auxin-treated explants. The work provides further experimental evidence on $L E C$ gene involvement in the embryogenic response in Arabidopsis somatic cells, and also implicates LEC1 function in more advanced stages of SE culture in relation to somatic embryo differentiation and development.
\end{abstract}

Keywords Auxin - Embryogenic transition - FUSCA3 . Hormonal mutants · LEAFY COTYLEDON1, LEC2 overexpression
Abbreviations
2,4-D 2,4-dichlorophenoxyacetic acid
E Induction medium
IZE Immature zygotic embryo

\footnotetext{
A. Ledwoń · M. D. Gaj ( $)$

Department of Genetics, University of Silesia,

Jagiellonska 28, 40-032 Katowice, Poland

e-mail: mmdgaj@us.edu.pl
}

SE Somatic embryogenesis

ZE Zygotic embryogenesis

\section{Introduction}

Somatic embryogenesis (SE) manifests a unique ability of plant cells to reprogram the normal pattern of somatic development into an embryogenic one, leading to the formation of structures resembling zygotic embryos. The SE process therefore provides an attractive system for studies on totipotency mechanisms and the developmental switch of somatic cells towards embryogenesis. Moreover, in plant biotechnology the phenomenon of SE is widely applied for micropropagation and genetic transformation.

The availability of a rich resource of genomic data, plus a number of versatile molecular tools, coupled with a simple in vitro culture system, predisposes Arabidopsis to molecular studies on SE (Gaj 2004). In addition, the implementation of genomic expression methods in the recent decade has greatly speed up the identification of numerous genes with potential involvement in the determination of SE. With the use of transcriptome analysis, candidate SE-specific genes were identified in a number of plant species, including gymnosperms (Stasolla et al. 2004; Aquea and Arce-Johnson 2008), Glycine max (ThibaudNissen et al. 2003), Triticum aestivum (Singla et al. 2007), cotton (Zeng et al. 2006), maize (Che et al. 2006), potato (Sharma et al. 2008), oil palm (Low et al. 2008) and Cyclamen persicum (Hoenemann et al. 2010). The available data confirmed a substantial complexity of gene networks involved in the onset and progress of SE (Zeng et al. 2007), in which genes with regulatory functions, such as transcription factors (TFs), are predominantly represented 
and predicted to have an essential role (Singla et al. 2007). However, the great majority of these genes needs further analysis to annotate their function in embryogenic development taking place in plant somatic cells. Only a small set of TF-specific genes have been experimentally proven to function in this way, including $B A B Y B O O M$ ( $B B M$, Boutilier et al. 2002), WUSCHEL (WUS, Zuo et al. 2002), AGAMOUS-LIKE15 (AGL15, Harding et al. 2003), and LEAFY COTYLEDON2 (LEC2) (Stone et al. 2001; Ledwoń and Gaj 2009).

Aside from $L E C 2$, two other $L E C$ genes, $L E C 1$ and FUS3, master regulators of the morphogenic and maturation phase of ZE (Harada 2001), are believed to be involved in SE. The $L E C$ genes are postulated to link the maturation phase of $\mathrm{ZE}$ and initiation of $\mathrm{SE}$ via the establishment of the proper environment for cellular differentiation (Braybrook and Harada 2008). In the context of SE process, $L E C 2$ is the best recognized regulator in terms of possible mechanisms involved in the reprogramming of somatic cells towards embryogenesis (Stone et al. 2001; Braybrook et al. 2006; Stone et al. 2008; Ledwoń and Gaj 2009).

LECI and FUS3 on the other hand are less well known in their role in SE. LEC1 and FUS3 proteins are built up from distinctly different structural domains. LEC1 contains a homolog of yeast HAP3, or the mammalian NF-YB/CBF-A trimeric CCAAT-binding factor subunit, $\mathrm{CBF}$, which consists of three domains (Lotan et al. 1998). During carrot embryo development C-LEC1 was indicated to be a component of the CCAAT box-binding complex together with C-HAP2B and C-HAP5A or C-HAP5B (Yazawa and Kamada 2007). LEC1, and a closely related LEC1-LIKE (L1L), constitute a class of LEC1- LIKE (L1L) proteins (Kwong et al. 2003). In a similar way to $L E C 1, L 1 L$ is expressed during seed development, but suppression of $L 1 L$ expression resulted in a phenotype different to the lecl mutant suggesting that LEC1 and L1L play quite different roles in embryogenesis (Kwong et al. 2003). In ZE LEC1 expression is referred to as embryo maturation (Meinke et al. 1994; West et al. 1994; Parcy et al. 1997), although activity of the gene has also been reported at earlier stages of zygotic embryo development (Wang et al. 2007). Knowledge on the molecular action of $L E C l$ and its target genes is limited. $L E C 1$ was implicated in seed storage protein accumulation via control of $F U S 3$ and $A B I 3$ expression (Kagaya et al. 2005a), and the regulation of $L E C 2$ by $L E C 1$, in a rather indirect manner, was suggested by Kagaya et al. 2005a and Santos-Mendoza et al. 2005. Recently, it was shown that LEC1, together with L1L, can activate CRUCIFERIN C (CRC), a seed storage protein, and SUCROSE SYNTHASE 2 (SUS2) via functional coupling with the ABA-response element (ABRE) binding factor (Yamamoto et al. 2009).
FUS3, together with $\mathrm{ABI} 3$ and LEC2 belongs to a AFL B3 subfamily of the plant-specific B3 domain of TFs, and its involvement in the transition between embryogenesis and vegetative phase of development, as was strongly proposed by Suzuki and McCarty (2008). These authors revealed the extensive role of the AFL B3 genes in regulating the principle hormone signaling pathways in seeds, including ABA, GA and auxin. Accordingly, FUS3 negatively regulates GA accumulation through repression of the GA-biosynthesis genes, GA3ox1 and GA3ox2 (Gazzarrini et al. 2004, Curaba et al. 2004), and up- regulates ABA accumulation (Gazzarrini et al. 2004). In a similar way to other LEC genes, FUS3 controls the synthesis of storage proteins and lipids during the maturation phase of $\mathrm{ZE}$ (Kagaya et al. 2005b), and the gene was found to be activated by LEC1 (Kagaya et al. 2005a) and LEC2 (Stone et al. 2008).

In plant development $L E C 1$ and FUS3 expression is limited to zygotic embryos, and declines during germination (Lotan et al. 1998; Reidt et al. 2001). The tight and multidirectional interactions between LEC1, FUS3 and $L E C 2$ genes during different stages of $\mathrm{ZE}$ has been documented to include a superior function of $L E C 1$ and $L E C 2$ over FUS3 (Wang et al. 2007) and mutual regulation of LEC1 and LEC2 (Santos-Mendoza et al. 2005; Kagaya et al. 2005a). It has not yet been elucidated whether similar interactions between $L E C$ genes exist in somatic cells induced to undergo embryogenesis.

In the present work, to gain more insight into the possible functions of LEC genes in SE, the expression profiles of $L E C l$ and FUS3 at various stages of in vitro induced embryogenic culture were defined, in relation to exogenous auxin treatment and tissue capacity for SE.

\section{Materials and methods}

\section{Plant material}

The Columbia (Col-0) genotype of Arabidopsis thaliana (L.) Heynh., and two hormonal mutants (axr4-1 and $\operatorname{cbp} 20$ ) of low capacity to produce somatic embryos under in vitro conditions (Gaj et al. 2006) were studied. The axr4-1 form belongs to a class of auxin resistant mutants (Hobbie and Estelle 1995), while cbp20 is hypersensitive for abscisic acid and is drought tolerant (Papp et al. 2004). Seeds of the axr4-1 mutant and Col-0 parental genotype were supplied by NASC (The Nottingham Arabidopsis Stock Centre), and the $\operatorname{cbp} 20$ mutant was kindly provided by Z. Szweykowska-Kulińska, University of Adam Mickiewicz-Poland. The cbp20 mutant carries a knock-out mutation (Papp et al. 2004) and the axr4-1 possesses a T-DNA insertion at the beginning of the AXR4 gene 
(Dharmasiri et al. 2006). Additionally, the Col-0 transgenic line $(12 / 1 / 8)$ harboring a single copy of the 35S::LEC2-GR construct, and expressing a high and stable level of $L E C 2$ transcript (Ledwoń and Gaj 2009), was also used in the experiments.

Somatic embryogenesis induced in vitro

Immature zygotic embryos (IZEs) with fully developed green and bent cotyledons were cultured in vitro following a standard protocol which enables efficient and direct somatic embryo development in the control Col-0 genotype (Gaj 2001). Ten IZEs were cultured in a Petri dish $(35 \mathrm{~mm})$ on Phytagel-solidified $\left(3.5 \mathrm{~g}^{-1}\right)$ induction medium (E) containing basal B5 micro and macro-elements (Gamborg et al. 1968) and $20 \mathrm{~g} \mathrm{l}^{-1}$ sucrose. E media with auxin, $5 \mu \mathrm{M}$ 2,4-D, Sigma (E5) and without auxin (E0) were used.

Plant growth and in vitro culture conditions

Plants used as a source of explants for in vitro culture were grown in mixture of the soil and vermiculite $(1: 1)$, at $22^{\circ} \mathrm{C}$ under a $16 \mathrm{~h}$ photoperiod of $100 \mu \mathrm{M} \mathrm{m}^{-2} \mathrm{~s}^{-1}$ white fluorescent light; and embryogenic cultures were kept at $23^{\circ} \mathrm{C}$ under a $16 \mathrm{~h}$ photoperiod of $40 \mu \mathrm{M} \mathrm{m}^{-2} \mathrm{~s}^{-1}$ white fluorescent light.

Induction of LEC2 protein activity in transgenic plants

To induce activity of the LEC2 protein a synthetic glucocorticoid, dexamethasone (water soluble DEX, Sigma), was added to the media at a concentration of $30 \mu \mathrm{M}$, as described by Ledwoń and Gaj (2009).

\section{RNA isolation and qRT-PCR analysis}

An RNAqueous Kit (AMBION) was used to isolate RNA. The concentration and purity of RNA was evaluated with a ND-1000 spectrophotometer (NanoDrop). To control for DNA contamination RNAs were treated with RQ1 RNasefree DNase I (Promega), following the manufacturer's instructions. First-strand c-DNA was produced in a $40 \mu \mathrm{l}$ reaction volume using the RevertAid First Strand cDNA Synthesis Kit (Fermentas). The product of reverse transcription was diluted with water in 1:1 ratio, and $1 \mu \mathrm{l}$ of this solution was used for quantitative RT-PCR (qRTPCR). qRT-PCR was done in a $10 \mu \mathrm{l}$ reaction volume with the use of LightCycler FastStart DNA Master SYBR Green I (Roche) and appropriate primers.

The following primers were used for qRT-PCR reactions of the analysed genes:
LEC1 (R): 5'-CTGGACCACGATACCATTGTT-3' (F): 5'-GTGGAGCTCCCTTCTCTCACT- $3^{\prime}$

FUS3 (R) TGAAGGTCCAAACGTGAAAAC (F):GTCAGCTCTCTCCGACGTATG

At4g27090 (R) 5'-CCTCGATCAAAGCCTTCTTCT-3'; (F) $5^{\prime}$-F:GTCGTTATCGTCGACGTTGTT- $3^{\prime}$

The LightCycler 2.0 (Roche) real-time detection system was used with the following reaction conditions: denaturation one repeat of $10 \mathrm{~min}$ at $95^{\circ} \mathrm{C}$, followed by 45 repeats of $10 \mathrm{~s}$ at $95^{\circ} \mathrm{C}, 8 \mathrm{~s}$ at $55^{\circ} \mathrm{C}, 12 \mathrm{~s}$ at $72^{\circ} \mathrm{C}$ and $5 \mathrm{~s}$ at $80^{\circ} \mathrm{C}$. Denaturation for melt curve analysis was conducted at $95^{\circ} \mathrm{C}$ followed by $15 \mathrm{~s}$ at 65 and $95^{\circ} \mathrm{C}\left(0.1^{\circ} \mathrm{C} / \mathrm{s}\right.$ for fluorescence measurement).

Primary data analysis was performed with LightCycler Software 4.0 (Roche). Relative RNA levels were calculated and normalized to an internal control, the At4g27090 gene encoding 60S ribosomal protein (Thellin et al. 1999). In all analyzed tissue samples the control gene exhibited a constant expression pattern with $\mathrm{Cp}=18 \pm 1$.

The expression values normalized to the internal control were standardized differently as indicated in the figure legends.

Total RNA was isolated from freshly isolated (0 day) explants induced on E5 and E0 media. Tissue sampling included 1, 3, 5, 10, 15 and 30 day for E5-induced cultures, and 3, 5 and 10 day for E0-growing explants. Depending on culture age, 250 (0 day)-2 (30 day) tissue samples were used per treatment. The plant tissues for the qRT-PCR analysis were produced in 3-4 independent biological replicates, and 2 technical replicates of each repetition were carried out.

Statistical analysis

ANOVA rang Kruskal-Wallis and U Mann-Whitney's statistical tests were applied to calculate significant differences (at $P=0.05$ ) between samples.

\section{Results}

Col-0 embryogenic culture

The analysis of LECland FUS3 expression profile took place during the time course of embryogenic cultures induced in vitro on auxin and auxin-free medium. Under in vitro conditions inductive for SE in Arabidopsis, i.e. in cultures of Col-0 IZEs on E5 auxin medium, the explant tissues undergo successive morphological changes characteristic of early, transient and advanced stages of embryogenesis (Fig. 1). The Real-Time PCR analysis 

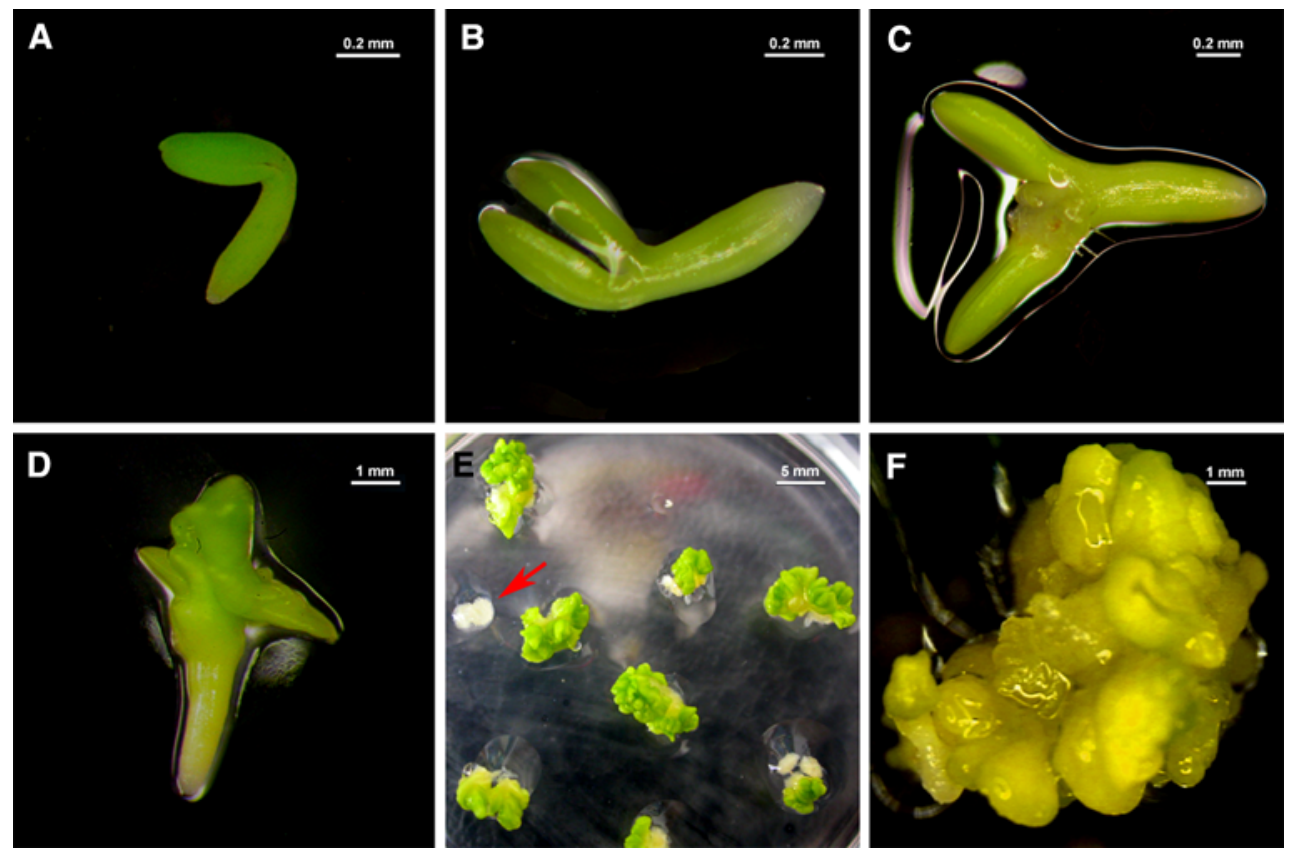

Fig. 1 Developmental stages of Col-0 IZE explants cultured on E5 medium (a-f) and analysed with Real-Time qPCR. a freshly isolated IZE explant of late cotyledonary stage ( 0 day); b straightening of cotyledons (3 day); c swelling and expanded cotyledons (5 day); d embryo-like protuberances on the surface of cotyledons (10 day); e explants covered with fully developed somatic embryos and callus (an arrow) formed sporadically from $10 \%$ of explants (15 day); f aging of culture and callus development (30 day) focused on selected stages of SE, and included: initiation phase ( 3 and 5 day-old cultures), manifested by distinct changes of cotyledon morphology (straightening, enlargement and swelling); emergence of the first embryos (10 day); development of numerous embryos (15 day) and aging of the cultures manifested by callus overgrowth due to the re-differentiation of somatic embryos. Beside embryogenic cultures induced on auxin medium, the IZEs induced on auxin-free medium (E0) were analysed up to day 10 , when instead of the embryogenic response, a regular development of seedlings is observed.

The expression level of the LECl gene in the tissues sampled between 0 and 30 day of Col-0 culture on E5 revealed a progressive expression pattern. A significant but temporary increase of expression was observed at the 1st day of culture, followed by a drastic decrease at 3 day and a subsequent successive increase of activity up to 15 day (Fig. 2a). In contrast to a period of embryogenic induction and intensive embryo development (3-15 day), related to stimulation of LEC1 activity, a significant fall of the gene activity was detected in aging 30 day-old cultures.

A distinctly different pattern of LECl expression was observed on auxin-free medium where, except for the 3rd day, the explants cultured on E0 medium presented up to sixfold lower (10 day) expression compared with auxininduced cultures (Fig. 2b). The observed differences in the pattern and level of expression found in E0 and E5 induced cultures demonstrates the SE-specific and auxin-mediated stimulation of LECl in embryogenic culture.

In order to further verify the suggestion that up-regulation of the LECl gene observed on auxin medium is specific to embryogenic tissue, the level of transcripts was also analysed in non-embryogenic Col-0 callus produced sporadically on $\mathrm{E} 5$ medium by some explants unable to undertake embryogenic transition. qRT-PCR analysis confirmed up to a tenfold lower expression level of LEC1 in 15 day callus compared with embryogenic cultures of the same age (Fig. 2c).

As for LEC1, the activity of FUS3 was detected at all stages of SE, but unlike LEC1 the expression level of FUS3 was not differentiated during $\mathrm{SE}$ and remained at the level observed in freshly isolated explants (Fig. 3a). Moreover, it was found that the expression profiles of FUS3 on E5 and E0 media were similar, implying that the gene activity is not influenced by exogenous auxin (Fig. 3b). Regardless of auxin presence in the medium, FUS3 transcripts displayed a steady level at the monitored stages of SE, suggesting that the gene activity is not specific to any particular stage, and that a higher expression level accompanies embryogenic cultures. Accordingly, FUS3 expression was increased in cultures producing somatic embryos above the level observed in nonembryogenic callus of Col-0 (Fig. 3c). 

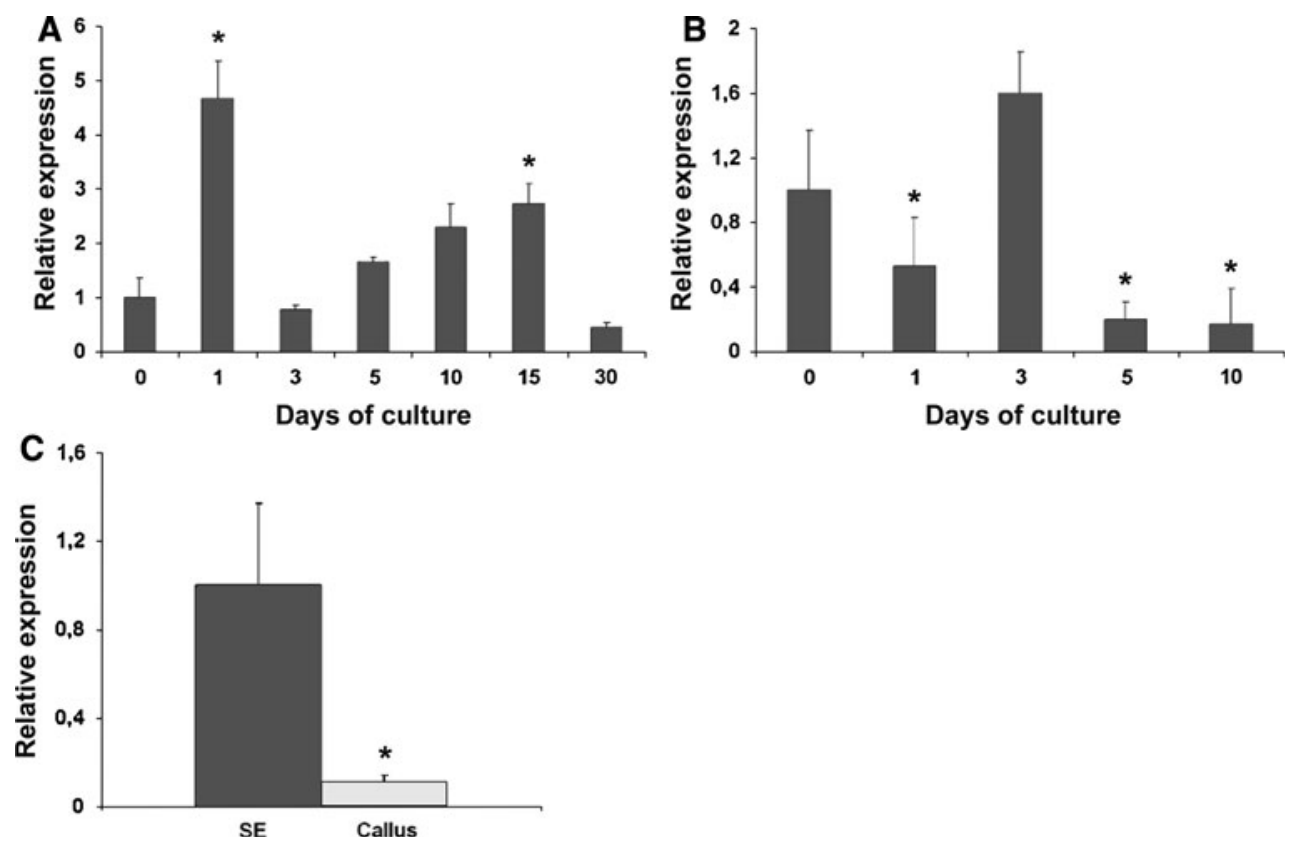

Fig. 2 Expression patterns of the $L E C 1$ gene in IZE-derived cultures. a embryogenic and (b) non-embryogenic cultures of Col-0 explants on auxin, E5 (a) and auxin-free, E0 (b) medium, respectively. c embryogenic (SE) and non-embryogenic (callus) tissue of Col-0 explants on E5 medium at 15 day. The activity of the gene was standardized to: E5

culture at 0 day (a), E5 culture (b), E5 embryogenic culture at 15 day (c). *Expression level significantly differs from those observed in: culture at 0 day (a); culture on E5 medium (b), embryogenic culture at 15 day (c); $P<0.05$. RNA levels were normalized to that of At4g27090. Bars show standard deviation
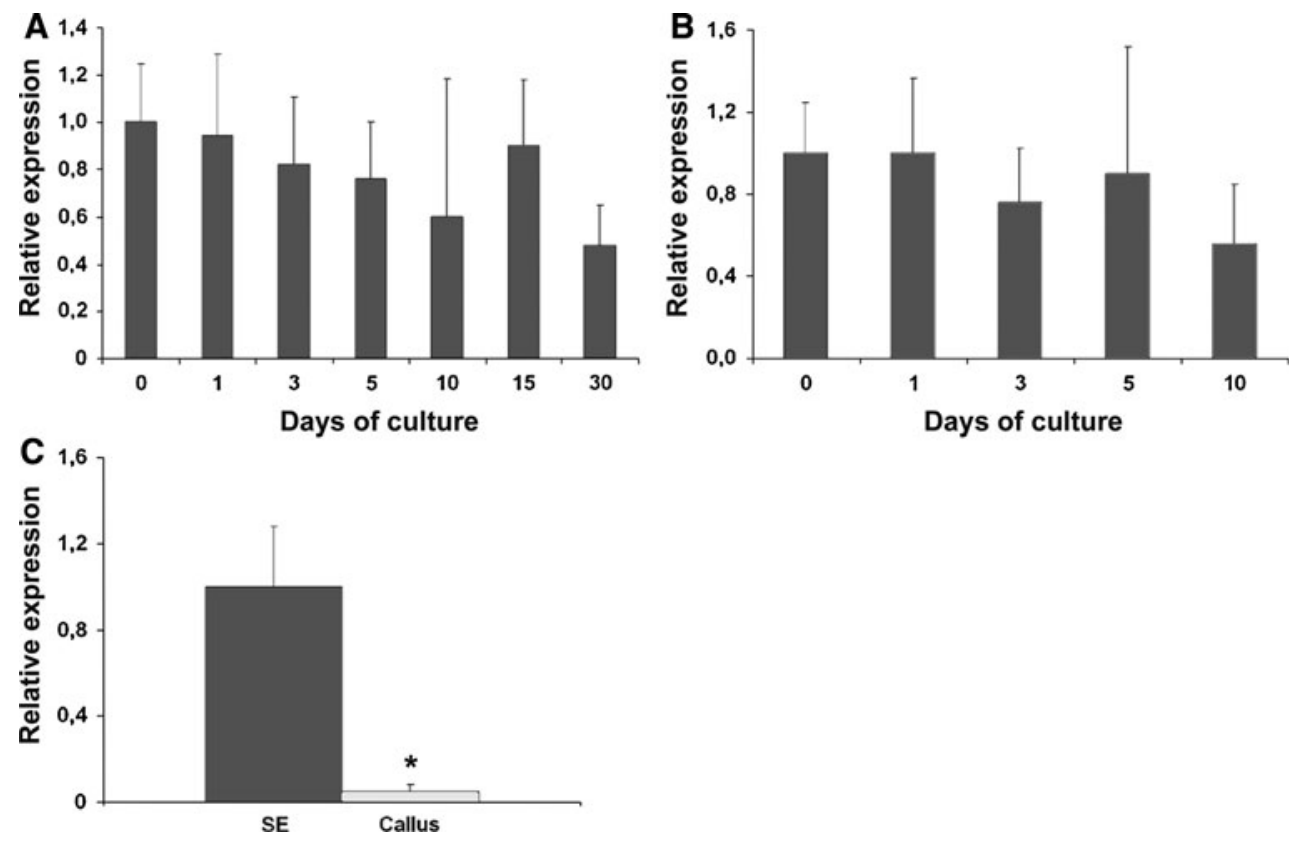

Fig. 3 Expression patterns of the FUS3 gene in IZE-derived cultures. (a) embryogenic and (b) non-embryogenic cultures of Col-0 explants on auxin, E5 (a) and auxin-free, E0 (b) medium, respectively. c embryogenic (SE) and non-embryogenic (callus) tissue of Col-0 explants on E5 medium at 15 day. The activity of the gene was

standardized to: E5 culture at 0 day (a), E5 culture (b), E5 embryogenic culture at 15 day (c). *Expression level significantly differs from those observed in: culture at 0 day (a); culture on E5 medium (b), embryogenic culture (c); $P<0.05$. RNA levels were normalized to that of At4g27090. Bars show standard deviation 


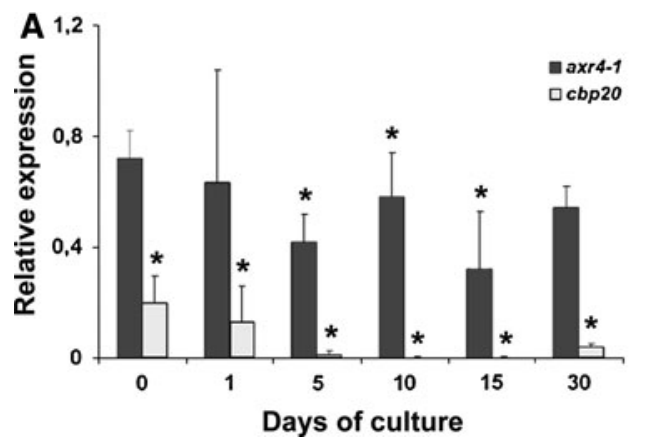

Fig. 4 Expression level of LEC1 (a) and FUS3 (b) genes in cultures of SE-impaired mutants (cbp20 and axr4-1) induced on E5 medium. The activity of the gene was standardized to Col-0 culture on E5. *Expression level differs significantly from observed in Col-0

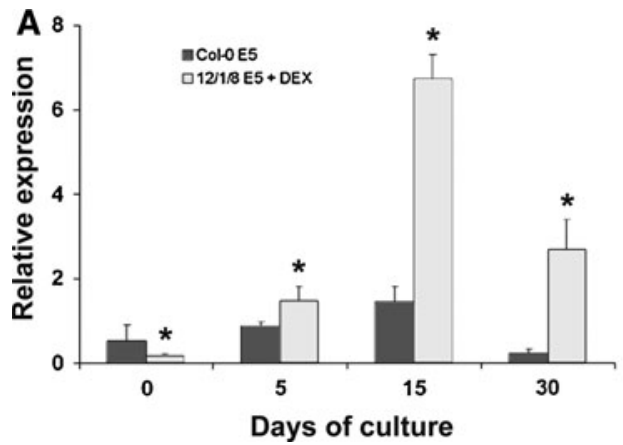

Fig. 5 Expression pattern of LEC1 (a) and FUS3 (b) genes in embryogenic cultures induced on E5 medium in explants overexpressing the LEC2 gene (35S::LEC2-GR, Col-0). The activity of the gene was standardized to Col-0 culture on E5. *Expression level

\section{Mutants impaired in SE capacity}

In addition to the Col-0 highly embryogenic genotype, LEC1 and FUS3 expression was also determined in cultures of $c b p 20$ and axr4-1 hormonal mutants (Fig. 4). The mutants were selected for their strongly impaired capacity for SE, which results in a decreased frequency of explants producing somatic embryos and abundant callus formation (Gaj et al. 2006). Both mutant cultures displayed patterns and levels of LEC1 (Fig. 4a) and FUS3 (Fig. 4b) expression, manifested by a significant reduction of their activity, different to Col-0. In $c b p 20$ there was an especially strong inhibition of gene activity, with highly reduced SE-efficiency and productivity (Gaj et al. 2006). This mutant also displayed a drastic decrease in $L E C l$ transcript level in all monitored time points of the culture, including freshly isolated explants, while FUS3 transcripts were significantly down-regulated from day 5 onwards.

\section{Embryogenic culture overexpressing LEC2}

The activity of the LECI and FUS3 genes was also monitored during a $0-30$ day period of embryogenic culture

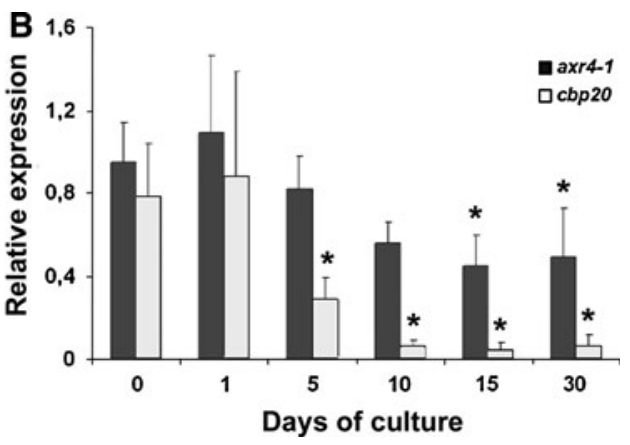

embryogenic culture $(P<0.05)$. RNA levels were standardized to embryogenic Col-0 culture and normalized to that of At4g27090. Bars show the standard deviation

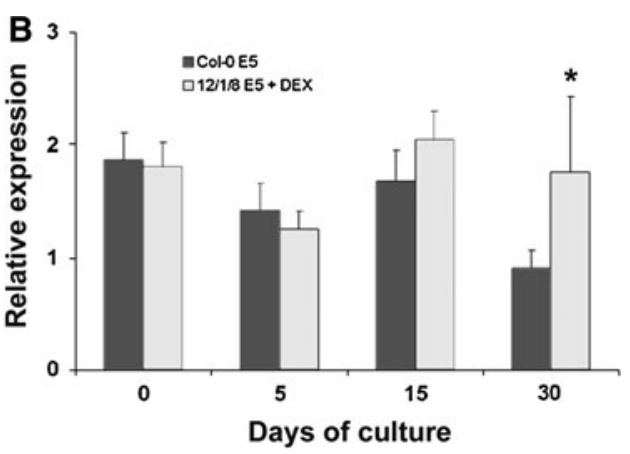

differs significantly from observed in Col-0 embryogenic culture $(P<0.05)$. RNA levels were normalized to that of At4g27090. Bars show standard deviation

derived from IZEs of the 35S::LEC2-GR transgenic line. The transgene encodes a fusion LEC2-GR protein which enters a nucleus only upon DEX treatment (Sablowski and Meyerowitz 1998). We proved a constitutive and highly increased LEC2 expression level (up to 3000-fold) in transgenic plants and in vitro cultured explants regardless of DEX treatment. Simultaneously, DEX treatment induced developmental abnormalities in transgenic seedling and somatic embryo development in planta and in vitro (Ledwoń and Gaj 2009). Moreover, functionality of the construct was also indicated by observation that DEX treatment induced expression of LECl and FUS3 in 7- and 28-day old transgenic plants (data not presented).

Analysis of LEC1 and FUS3 transcripts indicated that their general expression pattern in transgenic culture followed that observed in the Col- 0 control. Accordingly, a significant increase of $L E C l$ (Fig. 5a), and a constant FUS3 (Fig. 5b) expression level between 5 and 15 day of culture, were detected. However, under LEC2 overexpression a significant up-regulation (2-11-folds) of the LEC1 transcription level was recorded in cultures induced to initiate embryos. Contrary to $L E C 1$, expression level of FUS3 was not elevated in transgenic culture within a period 
corresponding to SE induction and embryo development. However, a significant up-regulation of FUS3 over the level detected in non-transgenic culture was observed in aging 30 day tissue.

\section{Discussion}

In agreement with an early postulate on the genetic similarity between somatic and zygotic embryogenesis (Zimmerman 1993), there is growing evidence confirming a close relationship between these apparently different embryogenic pathways triggered in two different cell types. Among the key genes controlling embryo development in zygotic and somatic plant cells, the LEC genes of Arabidopsis were postulated to play a crucial role (Braybrook and Harada 2008). The mechanisms involved in LECrelated control of $\mathrm{ZE}$ have been extensively investigated and recognized (Harada 2001; Braybrook and Harada 2008; Suzuki and McCarty 2008), while the function of $L E C$ in SE was already revealed. Recent experimental data strongly confirmed the link between the LEC2 gene and the induction of SE (Stone et al. 2008; Ledwoń and Gaj 2009), but much less is known about two other closely related LEC genes, LEC1 and FUS3, in terms of molecular mechanisms driven by them in somatic cells.

The suggestion on the involvement of LECI and FUS3 in SE are based on the following observations: (1) mutants of these genes lacking activity could not bring about effective SE induction (Gaj et al. 2005) and (2), LEC1 overexpression resulted in spontaneous somatic embryo formation in planta (Lotan et al. 1998). Moreover, the expression of $L E C 1$, and the closely related to LEC1-LIKE $(L 1 L)$ ortholog, during in vitro induced $\mathrm{SE}$, has been reported in numerous plants, including maize (Zhang et al. 2002), carrot (Yazawa et al. 2004), Helianthus annuus (Fambrini et al. 2006), Medicago sativa (Domoki et al. 2006), Theobroma cacao (Alemanno et al. 2008) and Vitis vinifera (Schellenbaum et al. 2008; Maillot et al. 2009). LEC1 expression was also found to be associated with ectopic formation of somatic embryos induced by overexpression of LEC2 (Stone et al. 2001) and MYB118 (Wang et al. 2009).

The present work has provided further evidence of a close relation between the embryogenic potential of tissues and the activity of LECI and FUS3. Real-time qRT-PCR analysis proved a high level of activity of $L E C I$ and FUS3 in Arabidopsis embryogenic culture, and established that their transcript level is significantly increased in highly embryogenic cultures compared with cultures impaired in SE.

The activity of LECI and FUS3 was found in all monitored stages of embryogenic cultures, but the distinctly different expression pattern displayed by the genes suggests their likely disparate functions in SE. LECl showed a differential expression pattern over the time course of SE, while FUS3 activity was constant at all monitored stages. The expression profile of $L E C l$ observed in Arabidopsis embryogenic culture suggests the involvement of the gene in the process of differentiation and development, rather than induction of somatic embryos; although a drastic increase of $L E C l$ activity was observed in the first day of culture. However, the transient character of this up-regulation indicates that general stress, not specific to SE, but imposed by in vitro conditions, is responsible for this stimulation, as was also observed in Arabidopsis for $L E C 2$ (Ledwoń and Gaj 2009). Embryogenic culture stress-related stimulation of $L E C 1$, and some other SE-associated genes, at the first day of embryogenic culture has also been reported in alfalfa (Domoki et al. 2006). The SE-related expression of $L E C 1$ revealed in the present work indicated a progressive and auxin-dependent increase in gene transcript level, which peaked at day 15 of embryogenic culture i.e. at a stage relevant to efficient development of somatic embryos. Consistent with Arabidopsis, in carrot (Yazawa et al. 2004) and alfalfa (Domoki et al. 2006) embryogenic cultures, $L E C 1$ expression reached maximum in advanced cultures efficiently producing somatic embryos in callus tissue. Considering these observations $L E C l$ is likely to be involved in somatic embryo differentiation and development. However, the possible function of $L E C 1$ in initiation of somatic embryos cannot be excluded, due to the spontaneous formation of somatic embryos in planta under its overexpression (Lotan et al. 1998). Accordingly, in a recent network of interacting LEC factors LEC1 was positioned very early during seed development, and upstream of the B3 network (Suzuki and McCarty 2008). A possible indirect link between $L E C l$ and the induction of embryogenesis can be expected in the light of a recent postulate that in ZE processes $L E C l$ may be activated by the presumptive carboxypeptidase ALTERED MERISTEM PROGRAM 1 $(A M P 1)$ involved in a general differentiation-promoting activity (Suzuki et al. 2008). Interestingly, the mutation in AMP1 results in higher capacity for SE manifested by embryo production in seedling culture (Mordhorst et al. 1998), and the recent finding that AMP1 interacts with the MP/ARF5 auxin response factor (Vidaurre et al. 2007) provides a new perspective on a possible links between auxin, $L E C l$ and embryogenesis.

In terms of the predicted $L E C I$ and auxin relationship, our work documents that expression of $L E C l$ during SE is auxin-stimulated. Similarly, in alfalfa embryogenic culture, up-regulation of MsLECl gene upon auxin treatment has also been demonstrated (Domoki et al. 2006). Furthermore, it was recently indicated that in sunflower, overexpression of a closely related LEAFY COTYLEDONI-LIKE (Ha-L1L) 
marked the putative founder cells of ectopic embryos, and co-localized with IAA accumulation (Chiappetta et al. 2009). These observations are consistent with auxin promoted activity of $L E C l$ postulated in studies on the turnip, a gain-of-function mutation of $L E C l$, leading to altered embryogenic response (Casson and Lindsey 2006). Thus it seems conceivable that $L E C l$ acts in concert with auxin to potentiate embryogenic pathways in planta and in vitro, and further analysis is needed to reveal other interacting factors, among which AMP1 may play an important role (Suzuki et al. 2008).

Besides auxin-related factors, ABA-controlled proteins are also expected to interplay with LEC1 during embryogenesis; and in this respect an interaction between LEC1/L1L and a seed-specific ABA-response element (ABRE)-binding factor, bZIP67, was recently reported in Arabidopsis seed development (Yamamoto et al. 2009). Interestingly, bZIP67 was also found to be expressed in embryogenic culture induced in vitro in Arabidopsis (M. Kurczyk, M. Gliwicka, MDG, unpublished data).

In addition to $L E C 1$ activity, the present analysis also indicates expression of FUS3 in Arabidopsis embryogenic cultures, as previously reported (Ikeda-Iwai et al. 2002; Gaj et al. 2005). However, it was found that contrary to LEC1 and LEC2 (Ledwoń and Gaj 2009) the level of FUS3 expression is constant, and not related with exogenous auxin treatment. Although the activity of the FUS3 gene does not seem to be related with any particular SE stage, its activity proved to be specific to embryogenic cultures. According to this, in Arabidopsis explants induced in vitro on cytokinin medium for adventitious shoot regeneration, a progressive decline of FUS3 expression was observed (M. Wajand, AL and MDG, unpublished), and the loss-offunction fus 3 mutant was found to be highly effective in shoot regeneration (Gaj et al. 2005).

During ZE, in contrast to SE, a differential expression pattern of FUS3 was described. Transcripts were not detected at early stages of zygote development, and the gene expression was indicated from the second week after fertilization up to the dry seed stage (Kroj et al. 2003; Baumbusch et al. 2004; Wang et al. 2007). Expression of FUS3 at the very early stages of SE, found here, can reflect the gene activity observed in explant tissue, the immature zygotic embryos at the late developmental stage used to induce embryogenesis, and seems not to be related with the gene involvement in SE inductive mechanisms. Moreover, considering that in ZE FUS3 activity is proposed to be regulated by $L E C 1$ (Kagaya et al. 2005a), its transcription during SE can result from its auxin-stimulated expression found in embryogenic culture. Interestingly, the present analysis indicates that auxin treatment was shown not to influence FUS3 expression level in cultured explants. In contrast to SE, in zygotic embryos FUS3 activity monitored with GUS was reported to be up-regulated by exogenous auxin (Gazzarrini et al. 2004). Thus, the possible link between FUS3 and auxin in zygotic and somatic cells remain unclear, and further studies are needed to elucidate the mechanism.

The activity of FUS3 in embryogenic culture was found to be different to that observed in planta, as well as in terms of its interactions with $L E C 2$. It has been shown that as a consequence of overexpression of $L E C 2$, two other LEC genes, LEC1 and FUS3 were induced in planta in vegetative organs (Santos-Mendoza et al. 2005; Stone et al. 2008). Recently, it has also been postulated that this LEC2-driven expression is mediated by auxin, as elevated production of this hormone can be expected under $L E C 2$ overexpression (Stone et al. 2008). In the present work, however, it was observed that in cultures of 35S::LEC2GR explants the increased activity of $L E C 1$, but not FUS3, was induced over a time course related to induction, differentiation and development of somatic embryos. Given up-regulation of FUS3 by LEC2, as indicated in ZE (Kroj et al. 2003; To et al. 2006), and included in a recently proposed network of interactions between LEC (Suzuki and McCarty 2008; Braybrook and Harada 2008), a constant FUS3 expression level, not influenced by $L E C 2$ expression during SE, suggests that complex and cell type-dependent relationships involving $L E C$ genes can be expected.

Taken all together, the present results on expression patterns of $L E C 1$ and FUS3 in embryogenic culture provide further evidences that these genes, together with $L E C 2$ (Ledwoń and Gaj 2009), determine embryogenic potential in Arabidopsis tissue. Discussing the possible mechanisms used by $L E C 1$ and FUS3 in determination/enhancing of embryogenic capacity in somatic cells, the well recognized links between $L E C$ genes and hormones such as auxin, GA and ABA (reviewed by Braybrook and Harada 2008), the key factors for SE induction (Jimenez 2005), are crucial. In support for this hypothesis, a significantly altered expression profile of LEC2 (Ledwoń and Gaj 2009) as well as LECI and FUS3 (the present work) was found in the cbp20 and axr4-1 hormonal mutants with impaired capacity for SE (Gaj et al. 2006). The cbp20 mutation disturbs abscisic acid signaling (Papp et al. 2004), possibly by influencing microRNAs involved in the regulation of developmental processes (Kim et al. 2008), while axr4-1 interferes with auxin influx in Arabidopsis cells (Dharmasiri et al. 2006). Thus, both mutations are expected to alter the hormonal environment of somatic cells, and to negatively interfere with their embryogenic capacity. How a significantly decreased $L E C$ expression level observed in the mutant explants and cultures is related with the $\operatorname{cbp} 20$ and $a x r 4-1$ mutations it remains to be revealed. 
Although the presented data strongly confirmed the key role of LEC factors in somatic embryogenesis in Arabidopsis, species-specific requirements in genetic mechanisms involved in embryogenic transition of somatic cells should also be considered. In this respect, in contrast to the recent data on the diverse effects caused by overexpression of $L E C$ genes documented in Arabidopsis, ectopic expression of LECl homolog gene in white spruce did not result in somatic embryo formation (Klimaszewska et al. 2010). Similarly, overexpression of $L E C 2$ and $B B M$ genes reported to induce SE in Arabidopsis plants (Stone et al. 2001; Boutilier et al. 2002) did not promote embryo development in tobacco (Rashid et al. 2007; Srinivisan et al. 2007).

\section{Conclusions}

Current advances in cellular and molecular mechanisms of SE in higher plants indicate the complexity of the factors involved in embryogenic transition, and among them the regulatory genes encoding transcription factors are of special interest (Yang and Zhang 2010). Within TFs, crucial for enabling embryogenic transition of somatic cells, the $L E C$ gene master regulators of $\mathrm{ZE}$, were indicated. Accordingly, in Arabidopsis, only the cells of immature zygotic embryo with high activity of $L E C$ genes are able to produce somatic embryos under in vitro culture. Differential and auxin-dependent expression of LEC1 (the present work) and LEC2 (Ledwoń and Gaj 2009) displayed during SE demonstrates their vital role in induction (LEC2), differentiation and development of somatic embryos (LECl). The activity of FUS3 was also indicated to be associated with SE, although the mechanisms which link the gene with capacity of somatic cells for embryogenesis are much less clear, and seem not to be directly related to auxin.

Given the key role of $L E C$ genes in defining embryogenic potential of somatic cells, a challenging perspective for controlling plant totipotency is associated with mechanisms of chromatin-based repression system, which restricts $L E C$ function to seed development (Zhang and Ogas 2009). To release embryogenic potential in any tissue of interest, the control of the key players essential for embryogenic transition must include TFs operating during SE and, on the other hand, the factors repressing their activity. Manipulation of the repressors of $L E C$ may provide a new approach for triggering embryogenic development in somatic cells ( $\mathrm{Su}-$ zuki et al. 2007; Tanaka et al. 2008).

Open Access This article is distributed under the terms of the Creative Commons Attribution Noncommercial License which permits any noncommercial use, distribution, and reproduction in any medium, provided the original author(s) and source are credited.

\section{References}

Alemanno L, Devic M, Niemenak N, Sanier C, Guilleminot J, Rio M, Verdeil JL, Montoro P (2008) Characterization of leafy cotyledon1-like during embryogenesis in Theobroma cacao L. Planta 227:853-866

Aquea F, Arce-Johnson P (2008) Identification of genes expressed during early somatic embryogenesis in Pinus radiate. Plant Physiol Biochem 46:559-568

Baumbusch LO, Hughes DW, Galau GA, Jakobsen KS (2004) LEC1, FUS3, $A B I 3$ and Em expression reveals no correlation with dormancy in Arabidopsis. J Exp Bot 55:77-87

Boutilier K, Offringa R, Sharma VK, Kieft H, Ouellet T, Zhang L, Hattori J, Liu CM, van Lammeren AAM, Miki BLA, Custers JBM, van Lookeren Compagne MM (2002) Ectopic expression of $B A B Y B O O M$ triggers a conversion from vegetative to embryonic growth. Plant Cell 14:1737-1749

Braybrook SA, Harada JJ (2008) LECs go crazy in embryo development. Trends Plant Sci 13:624-630

Braybrook SA, Stone SL, Park S, Bui AQ, Le BH, Fischer RL, Goldberg RB, Harada JJ (2006) Genes directly regulated by $L E A F Y$ COTYLEDON2 provide insight into the control of embryo maturation and somatic embryogenesis. Pro Natl Acad Sci USA 103:3468-3473

Casson SA, Lindsey K (2006) The turnip mutant of Arabidopsis reveals that LEAFY COTYLEDON1 expression mediates the effects of auxin and sugar to promote embryonic cell identity. Plant Physiol 142:526-541

Che P, Love TM, Frame BR, Wang K, Carriquiry AL, Howell SH (2006) Gene expression pattern during somatic embryo development and germination in maize Hi II callus cultures. Plant Mol Biol 62:1-14

Chiappetta A, Fambrini M, Petrarulo M, Rapparini F, Michelotti V, Bruno L, Greco M, Baraldi R, Salvini M, Pugliesi C, Bitonti MB (2009) Ectopic expression of LEAFY COTYLEDON1-LIKE gene and localized auxin accumulation mark embryogenic competence in epiphyllous plants of Helianthus annuus $3 \mathrm{H}$. tuberosus. Ann Bot 103:735-747

Curaba J, Moritz T, Blervaque R, Parcy F, Raz V, Herzog M, Vachon $\mathrm{G}$ (2004) AtGA3ox2, a key gene responsible for bioactive gibberellin biosynthesis, is regulated during embryogenesis by LEAFY COTYLEDON2 and FUSCA3 in Arabidopsis. Plant Physiol 136:3660-3669

Dharmasiri S, Swarup R, Mockaitis K, Dharmasiri N, Singh SK, Kowalchyk M, Marchant A, Mills S, Sandberg G, Bennett MJ, Estelle M (2006) AXR4 is required for localization of the auxin influx facilitator AUX1. Science 312:218-220

Domoki M, Györgyey J, Bíró J, Pasternak TP, Zvara A, Bottka S, Puskás LG, Dudits D, Fehér A (2006) Identification and characterization of genes associated with the induction of embryogenic competence in leaf-protoplast-derived alfalfa cells. Biochem Biophys Acta 1759:543-551

Fambrini M, Durante C, Cionini G, Geri C, Giorgetti L, Michelotti V, Salvini M, Pugliesi C (2006) Characterization of LEAFY COTYLEDON1-LIKE gene in Helianthus annuus and its relationship with zygotic and somatic embryogenesis. Dev Genes Evol 216:253-264

Gaj MD (2001) Direct somatic embryogenesis as a rapid and efficient system for in vitro regeneration of Arabidopsis thaliana. Plant Cell Tissue Organ Cult 64:39-46

Gaj MD (2004) Factors influencing somatic embryogenesis induction and plant regeneration with particular reference to Arabidopsis thaliana (L.) Heynh. Plant Growth Regul 43:27-47

Gaj MD, Zhang S, Harada JJ, Lemaux PG (2005) LEAFY COTYLE$D O N$ genes are essential for induction of somatic embryogenesis of Arabidopsis. Planta 222:977-988 
Gaj MD, Trojanowska A, Ujczak A, Mędrek M, Kozioł A, Garbaciak B (2006) Hormone-response mutants of Arabidopsis thaliana (L.) Heynh. impaired in somatic embryogenesis. Plant Growth Regul 49:183-197

Gamborg OL, Miller RA, Ojima K (1968) Nutrient requirement of suspension culture of soybean root cells. Exp Cell Res 50:151-158

Gazzarrini S, Tsuchiya Y, Lumba S, Okamoto M, McCourt P (2004) The transcription factor FUSCA3 controls development timing in Arabidopsis through the hormones gibberellin and abscisic acid. Dev Cell 7:373-385

Harada JJ (2001) Role of Arabidopsis LEAFY COTYLEDON genes in seed development. J Plant Physiol 158:405-409

Harding EW, Tang W, Nichols KW, Fernandez DE, Perry SE (2003) Expression and maintenance of embryogenic potential is enhanced through constitutive expression of AGAMOUS-Like15. Plant Physiol 133:653-663

Hobbie L, Estelle M (1995) The axr4 auxin-resistant mutants of Arabidopsis thaliana define a gene important for root gravitropism and lateral root initiation. Plant J 7:211-220

Hoenemann C, Richardt S, Kruger K, Zimmer AD, Hoheand A, Stefan AR (2010) Large impact of the apoplast on somatic embryogenesis in Cyclamen persicum offers possibilities for improved developmental control in vitro. BMC Plant Biol 10:77

Ikeda-Iwai M, Satoh S, Kamada H (2002) Establishment of a reproducible tissue culture system for the induction of Arabidopsis somatic embryos. J Exp Bot 53:1575-1580

Jimenez VM (2005) Involvement of plant hormones and plant growth regulators on in vitro somatic embryogenesis. Plant Growth Regul 47:91-110

Kagaya Y, Toyoshima R, Okuda R, Usui H, Hattori T (2005a) LEAFY COTYLEDON1 controls seed storage protein genes through its regulation of FUSCA3 and ABSCISIC ACID INSENSITIVE3. Plant Cell Physiol 46:399-406

Kagaya Y, Okuda R, Ban A, Toyoshima R, Tsutumida K, Usui H, Yamamoto A, Hattori T (2005b) Indirect ABA-dependent regulation of seed storage protein genes by FUSCA3 transcription factor in Arabidopsis. Plant Cell Physiol 46:300-311

Kim S, Yang JY, Xu J, Jang ICh, Progge MJ, Chua NH (2008) Two cap-binding proteins $\mathrm{CBP} 20$ and $\mathrm{CBP} 80$ are involved in processing primary microRNAs. Plant Cell Physiol 49:16341644

Klimaszewska K, Pelletier G, Overton C, Stewart D, Rutledge RG (2010) Hormonally regulated overexpression of Arabidopsis WUS and conifer LEC1 (CHAP3A) in transgenic white spruce: implications for somatic embryo development and somatic seedling growth. Plant Cell Rep 29:723-734

Kroj T, Savino G, Valon C, Giraudat J, Parcy F (2003) Regulation of storage protein gene expression in Arabidopsis. Development 130:6065-6073

Kwong RW, Bui AQ, Lee H, Kwong LW, Fischer RL, Goldberg RB, Harada JJ (2003) LEAFY COTYLEDON-LIKE defines a class of regulators essential for embryo development. Plant Cell 15:5-18

Ledwoń A, Gaj MD (2009) LEAFY COTYLEDON2 gene expression and auxin treatment in relation to embryogenic capacity of Arabidopsis somatic cells. Plant Cell Rep 28:1677-1688

Lotan T, Ohtom M, Matsudaira Yee K, West MAL, Lo R, Kwong RW, Yamagishi K, Fischer RL, Goldberg RB, Harada JJ (1998) Arabidopsis LEAFY COTYLEDON1 is sufficient to induce embryo development in vegetative cells. Cell 93:1195-1205

Low ET, Alias H, Boon SH, Shariff EM, Tan CY, Ooi LC, Cheah SC, Raha AR, Wan KL, Singh R (2008) Oil palm (Elaeis guineensis Jacq.) tissue culture ESTs: identifying genes associated with callogenesis and embryogenesis. BMC Plant Biol 8:62

Maillot P, Lebel S, Schellenbaum P, Jacques A, Walter B (2009) Differential regulation of SERK, LEC1-Like and Pathogenesis-
Related genes during indirect secondary somatic embryogenesis in grapevine. Plant Physiol Biochem 47:743-752

Meinke DW, Franzmann LH, Nickle TC, Yeung EC (1994) leafy cotyledon mutants of Arabidopsis. Plant Cell 6:1049-1064

Mordhorst AP, Voerman KJ, Hartog MV, Meijer EA, van Went J, Koornneef M, de Vries SC (1998) Somatic embryogenesis in Arabidopsis thaliana is facilitated by mutations in genes repressing meristematic cell divisions. Genetics 149:549-563

Papp I, Mur LA, Dalmadi A, Dulai S, Koncz C (2004) A mutation in Cap Binding Protein 20 gene confers drought tolerance to Arabidopsis. Plant Mol Biol 55:679-686

Parcy F, Valon Ch, Kohara A, Miséra S, Giraudat J (1997) The ABSCISIC ACID-INSENSITIVE3, FUSCA3 and LEAFY COTYLEDON1 loci act in concert to control multiple aspects of Arabidopsis seed development. Plant Cell 9:1265-1277

Rashid SZ, Yamaji N, Masaharu K (2007) Shoot formation from root tip region: a developmental alteration by WUS in transgenic tobacco. Plant Cell Rep 26:1449-1455

Reidt W, Ellerström TM, Kölle K, Tewes A, Tiedemann J, Altschmied L, Bäumlein H (2001) FUS3-depened gene regulation during late embryogenesis. J Plant Physiol 158:411-418

Sablowski RWM, Meyerowitz EM (1998) A homolog of NO APICAL MERISTEM is an immediate target of the floral homeotic genes APETALA3/PISTILLATA. Cell 92:93-103

Santos-Mendoza SM, Dubreucq B, Miquel M, Caboche M, Lepiniec L (2005) LEAFY COTYLEDON2 activation is sufficient to trigger the accumulation of oil and seed specific mRNAs in Arabidopsis leaves. FEBS Lett 579:4666-4670

Schellenbaum P, Jacques A, Maillot P, Bertsch Ch, Mazet F, Farine S, Walter B (2008) Characterization of VvSERK1, VvSERK2, $V v S E R K 3$ and $V v L 1 L$ genes and their expression during somatic embryogenesis of grapevine (Vitis vinifera L.). Plant Cell Rep 27:1799-1809

Sharma SK, Millam S, Hedley PE, McNicol J, Bryan GJ (2008) Molecular regulation of somatic embryogenesis in potato: an auxin led perspective. Plant Mol Biol 68:185-201

Singla B, Tyagi AK, Khurana JP, Khurana P (2007) Analysis of expression profile of selected genes expressed during auxininduced somatic embryogenesis in leaf base system of wheat (Triticum aestivum) and their possible interactions. Plant Mol Biol 65:677-692

Srinivisan C, Liu Z, Heidmann I, Supena EDJ, Fukuoka H, Joosen R, Lambalk J, Angenent G, Scorza R, Custers JBM, Boutilier K (2007) Heterologus expression of the BABY BOOM AP2/ERF transcription factor enhances the regeneration capacity of tobacco (Nicotiana tabacum L.). Planta 225:341-351

Stasolla C, Bozhkov PV, Chu TM, van Zyl L, Egertsdotter U, Suarez MF, Craig D, Wolfinger RD, von Arnold S, Sederoff RR (2004) Variation in transcript abundance during somatic embryogenesis in gymnosperms. Tree Physiol 24:1073-1085

Stone SL, Kwong LW, Yee KM, Pelletier J, Lepiniec L, Fischer RL, Goldberg RB, Harada JJ (2001) LEAFY COTYLEDON2 encodes B3 domain transcription factor that induces embryo development. Pro Natl Acad Sci USA 98:11806-11811

Stone SL, Braybrook SA, Paula SL, Kwong LW, Meuser J, Pelletier J, Hsieh TF, Fischer RL, Goldberg RB, Harada JJ (2008) Arabidopsis LEAFY COTYLEDON2 induces maturation traits and auxin activity: implications for somatic embryogenesis. Pro Natl Acad Sci USA 105:3151-3156

Suzuki M, McCarty DR (2008) Functional symmetry of the B3 network controlling seed development. Curr Opin Plant Biol 11:548-553

Suzuki M, Wang HH-Y, McCarty DR (2007) Repression of LEAFY COTYLEDON1/B3 regulatory network in plant embryo development by VPIIABSCISIC ACID INSENSITIVE3-LIKE B3 genes. Plant Physiol 143:902-911 
Suzuki M, Latshaw S, Sato Y, Settles AM, Koch KE, Hannah LC, Kojima M, Sakakibara H, McCarty DR (2008) The maize Viviparous 8 locus, encoding a putative AMP1-like peptidase, regulates $\mathrm{ABA}$ accumulation and coordinates embryo and endosperm development. Plant Physiol 146:1193-1206

Tanaka M, Kikuchi A, Kamada H (2008) The Arabidopsis Histone Deacetylases HDA6 and HDA19 contribute to the repression of embryonic properties after germination. Plant Physiol 146: 149-161

Thellin O, Zorzi W, Lakaye B, De Borman B, Coumans B, Hennen G, Grisar T, Igout A, Heinen E (1999) Housekeeping genes as internal standards: use and limits. J Biotechnol 75:291-295

Thibaud-Nissen F, Shealy RT, Khanna A, Vodkin LO (2003) Clustering of microarray data reveals transcript patterns associated with somatic embryogenesis in soybean. Plant Physiol 132:118-136

To A, Valon C, Savino G, Guilleminot J, Devic M, Giraudat J, Parcy F (2006) A network of local and redundant gene regulation governs Arabidopsis seed maturation. Plant Cell 18:1642-1651

Vidaurre DP, Ploense S, Krogan NT, Berleth T (2007) AMP1 and MP antagonistically regulate embryo and meristem development in Arabidopsis. Development 134:2561-2567

Wang H, Guo J, Lambert KN, Lin Y (2007) Developmental control of Arabidopsis seed oil biosynthesis. Planta 226:773-783

Wang X, Niu QW, Teng C, Li C, Mu J, Chua NH, Zuo J (2009) Overexpression of PGA37/MYB118 and MYB115 promotes vegetative-to-embryonic transition in Arabidopsis. Cell Res 19:224-235

West MAL, Yee KM, Danao J, Zimmerman JL, Fischer RL, Goldberg RB, Harada JJ (1994) LEAFY COTYLEDON1 is an essential regulator of late embryogenesis and cotyledon identity in Arabidopsis. Plant Cell 6:1731-1745
Yamamoto A, Kagaya Y, Toyoshima R, Kagaya M, Takeda S, Hattori T (2009) Arabidopsis NF-YB subunits LEC1 and LEC1-LIKE activate transcription by interacting with seed-specific ABREbinding factors. Plant J 58:843-856

Yang X, Zhang X (2010) Regulation of somatic embryogenesis in higher plants. Crit Rev Plant Sci 29:36-57

Yazawa K, Kamada H (2007) Identification and characterization of carrot HAP factors that form a complex with the embryo-specific transcription factor C-LEC1. J Exp Bot 58:3819-3828

Yazawa K, Takahata K, Kamada H (2004) Isolation of the gene encoding carrot Leafy Cotyledon1 and expression analysis during somatic and zygotic embryogenesis. Plant Physiol Biochem 42:215-223

Zeng F, Zhang X, Zhu L, Tu L, Guo X, Nie Y (2006) Isolation and characterization of genes associated to cotton somatic embryogenesis by suppression subtractive hybridization and macroarray. Plant Mol Biol 60:167-183

Zeng F, Zhang X, Cheng L, Hu L, Zhu L, Cao J, Guo X (2007) A draft gene regulatory network for cellular totipotency reprogramming during plant somatic embryogenesis. Genomics 90 : $620-628$

Zhang H, Ogas J (2009) An epigenetic perspective on developmental regulation of seed genes. Mol Plant 4:610-627

Zhang S, Wong L, Meng L, Lemaux PG (2002) Similarity of expression patterns of knotted 1 and ZmLEC1 during somatic and zygotic embryogenesis in maize (Zea mays L.). Planta 215:191-194

Zimmerman JL (1993) Somatic embryogenesis: a model for early development in higher plants. Plant Cell 5:1411-1423

Zuo J, Niu QW, Frugis G, Chua NH (2002) The WUSCHEL gene promotes vegetative-to-embryonic transition in Arabidopsis. Plant J 30:349-359 